\title{
Magnetic sensitivity mediated by the Arabidopsis blue-light receptor cryptochrome occurs during flavin reoxidation in the dark
}

\author{
Marootpong Pooam ${ }^{1} \cdot$ Louis-David Arthaut $^{1} \cdot$ Derek Burdick $^{1,2} \cdot$ Justin Link $^{2} \cdot$ Carlos F. Martino $^{3} \cdot$ Margaret Ahmad $^{1,2}$
}

Received: 30 April 2018 / Accepted: 29 August 2018 / Published online: 7 September 2018

(c) The Author(s) 2018

\begin{abstract}
Main conclusion Arabidopsis cryptochrome mediates responses to magnetic fields that have been applied in the absence of light, consistent with flavin reoxidation as the primary detection mechanism.
\end{abstract}

\begin{abstract}
Cryptochromes are highly conserved blue-light-absorbing flavoproteins which have been linked to the perception of electromagnetic stimuli in numerous organisms. These include sensing the direction of the earth's magnetic field in migratory birds and the intensity of magnetic fields in insects and plants. When exposed to light, cryptochromes undergo flavin reduction/reoxidation redox cycles leading to biological activation which generate radical pairs thought to be the basis for magnetic sensitivity. However, the nature of the magnetically sensitive radical pairs and the steps at which they act during the cryptochrome redox cycle are currently a matter of debate. Here, we investigate the response of Arabidopsis cryptochrome-1 in vivo to a static magnetic field of $500 \mu \mathrm{T}(10 \times$ earth's field) using both plant growth and light-dependent phosphorylation as an assay. Cryptochrome responses to light were enhanced by the magnetic field, as indicated by increased inhibition of hypocotyl elongation and increased cryptochrome phosphorylation. However, when light and dark intervals were given intermittently, a plant response to the magnetic field was observed even when the magnetic field was given exclusively during the dark intervals between light exposures. This indicates that the magnetically sensitive reaction step in the cryptochrome photocycle must occur during flavin reoxidation, and likely involves the formation of reactive oxygen species.
\end{abstract}

Keywords Blue-light photoreceptor $\cdot$ Cryptochromes $\cdot$ Magnetoreceptor $\cdot$ Photomorphogenesis $\cdot$ Radical Pairs $\cdot$ ROS

\begin{tabular}{ll}
\multicolumn{2}{l}{ Abbreviations } \\
cry & Cryptochrome \\
GMF & Geomagnetic field \\
MF & Magnetic field \\
phy & Phytochrome
\end{tabular}

Margaret Ahmad

margaret.ahmad@upmc.fr

1 Sorbonne Universités, CNRS, UMR8256, IBPS, Photobiology Research Group, 7 Quai St. Bernard, 75005 Paris, France

2 Xavier University, 3800 Victory Parkway, Cincinnati, OH 45207, USA

3 Department of Biomedical Engineering, Florida Institute of Technology, 150W University Blvd, Melbourne, FL 32901, USA

\section{Introduction}

Cryptochromes are highly conserved flavoprotein bluelight receptors implicated in numerous aspects of plant growth and development including seedling de-etiolation, response to stress, the initiation of flowering, leaf morphology, elongation growth, and the entrainment of the circadian clock (Chaves et al. 2011; Ozturk 2017; Yang et al. 2017). Structurally, cryptochromes are flavoproteins that undergo light-induced photoreduction, whereby a bound FAD cofactor is reduced from the dark-adapted, oxidized redox state (FADox) to the radical $\left(\right.$ FADH $\left.^{\circ}\right)$, and reduced (FADH-) redox states upon illumination. Formation of the reduced redox state has been correlated with biological activity in numerous studies (Banerjee et al. 2007; Bouly et al. 2007; Kondoh et al. 2011; see review of current literature in Ahmad 2016) as a consequence of conformational change leading to the active state. The reduced flavin cofactor is subsequently oxidized in the presence of molecular oxygen to restore the resting, dark-adapted stage in a reoxidation 
reaction that does not require light (Muller and Ahmad 2011). In sum, under conditions of illumination, the cryptochrome photoreceptors are constantly cycling between inactive (oxidized) and activated (reduced) redox states, such that the net biological activity results from the sum of the light-induced (activating) and reverse (de-activating) redox reactions at any given timepoint. A model of the cryptochrome photocycle incorporating these elements and an estimation of the quantum efficiency of redox state interconversions both in vitro and in vivo has been recently derived (Procopio et al. 2016).

Subsequent to their initial discovery in plants, cryptochromes were identified in numerous other organisms including in insects and vertebrates. These homologues are highly similar structurally and some (for instance, insect type I cryptochromes) have been shown to undergo similar redox reactions to plant cryptochromes and activated in a similar manner (Ganguly et al. 2016; Lin et al. 2018).

Of particular interest was the identification of cryptochromes in the UV cones of the avian retina, which are suitably positioned for magnetosensing in migratory birds, which use the geomagnetic field (GMF) for directional orientation (Wiltschko and Wiltschko 2012). The suggestion that cryptochromes may be magnetoreceptors in birds was originally put forward on the grounds that the redox cycle of cryptochromes can generate unpaired radicals, which on theoretical grounds may be affected by weak magnetic fields (MFs; see Hore and Mouritsen 2016 for review). Behavioral studies then showed that wavelengths of light corresponding to the cryptochrome flavin absorption spectrum are in fact correlated with avian magnetoreception, and that, furthermore, the cryptochrome protein in the avian retina undergoes conformational change compatible with the deduced redox cycle of cryptochromes (Nießner et al. 2013; Wiltschko and Wiltschko 2014). Thus, a model for magnetosensing has been suggested, whereby the rate constants (efficiency of redox state interconversion) of the cryptochrome redox cycle are altered in response to weak electromagnetic fields, with a resulting change in biological activity. Support for this suggestion has come from numerous studies in diverse organisms, where cryptochrome mutants are available. Using such mutants, it was possible to show that cryptochromes are required for effects of low-level MFs on growth and developmental responses in Arabidopsis (Ahmad et al. 2007; Xu et al. 2012, 2014, 2015) and on behavioral and physiological responses to low level applied MFs in Drosophila (e.g., Yoshii et al. 2009; Foley et al. 2011; Fedele et al. 2014; Marley et al. 2014; Wu et al. 2016).

The effects of applied MFs on Arabidopsis seedling growth and cryptochrome phosphorylation are relatively minor, and, indeed, require highly controlled conditions of blue-light intensity to visualize. It would, therefore, appear unlikely that Arabidopsis, or, indeed, plants, in general, would show a response to possible minor fluctuations or changes in direction of MFs in the natural environment. Nonetheless, geomagnetic fields (GMFs) have been suggested as a possible factor in plant evolution. During the Tertiary and Cretaceous periods, when Angiosperms diversified into many orders and families, there were also a number of reversals in GMF polarity accompanied by sharp decreases in GMF intensity. The possible influence of these significant changes in GMF has thereby been suggested to be involved in plant evolution (Maffei 2014; Occhipinti et al. 2014; Van der Straeten et al. 2018). Moreover, at significantly elevated MF levels (in the mTessla range), effects on germination and increased root length have been reported, as well as increase in oxidative stress (increased $\mathrm{H}_{2} \mathrm{O}_{2}$ levels and decrease in antioxidant activity) (see Maffei 2014 for review). Therefore, a possible role for magnetic sensitivity in plant development and evolution remains an open question.

In order for a biological photoreceptor to function as a magnetoreceptor, it must first produce unpaired radicals with suitable characteristics for interaction with applied MFs, as predicted by the radical-pair hypothesis (Hore and Mouritsen 2016). Cryptochromes are one of the rare classes of photoreceptor that can in fact form multiple such unpaired radicals during activation. They do so at several different steps in the course of flavin redox state interconversion, including both during forward (light-driven) flavin reduction and during reverse (light-independent) flavin reoxidation of the photocycle. As a result, an applied MF could be predicted to alter the chemical rate constants of these redox reactions, creating a difference in the concentration of the activated state receptor to result in altered biological activity.

At the present time, most attention has focussed on the forward (light-driven) electron transfer reaction of cryptochromes as a possible magnetically sensitive reaction. In this reaction, an electron is transferred to the oxidized flavin from the protein surface via a chain of intraprotein electron transfer comprising multiple tryptophan (Trp) and tyrosine (Tyr) residues (see Chaves et al. 2011 for review). This results in the formation of $\operatorname{Trp}^{\circ} / \mathrm{FADH}^{\circ}$ or $\mathrm{Tyr}^{\circ} / \mathrm{FADH}^{\circ}$ radicals which have shown suitable characteristics in modelling studies for interaction with weak MFs and thereby alter the rate constants for redox state interconversion (Hore and Mouritsen 2016). In support of this hypothesis, experimental evidence with isolated Arabidopsis and Drosophila cryptochromes as well as the very similar E. coli photolyase has shown magnetic effects on forward electron transfer reaction rates in vitro, albeit only at relatively high MF strength (in the $\mathrm{mT}$ range) (Maeda et al. 2012; Sheppard et al. 2017).

However, a number of recent studies in the avian directional sensing system are in contradiction to this view. First, it has been demonstrated that robins are able to orient to the geomagnetic field (GMF) in green light, at a wavelength that can only be absorbed by the $\mathrm{FADH}^{\circ}$ neutral radical redox 
state of cryptochrome. In consequence, the flavin radical would be fully reduced in the course of the reaction (from $\mathrm{FADH}^{\circ}$ to FADH-) and there is no possibility for a $\operatorname{Trp}^{\circ}$ or $\mathrm{Tyr}^{\circ} / \mathrm{FADH}^{\circ}$ radical pair to be formed and undergo modification by the MF (Nießner et al. 2013). Even more telling was the result from so-called 'flicker' experiments, where birds were subjected to intermittent pulses of light and dark during the behavioral experiments (Wiltschko et al. 2016). In these tests, the birds were exposed to MF direction only during the dark interval between light pulses. The birds in fact proved to be directionally oriented under these conditions. Since a $\operatorname{Trp} \%$ flavin or $\operatorname{Tyr}^{\circ} /$ flavin radical pair has a very short lifetime (few milliseconds), these radicals could not be the targets for the MF and, therefore, could not be implicated in magnetosensing. These experiments instead indicated that the magnetosensitive step in the bird orientation experiments must occur in the dark period, which concurs with the reoxidation reaction of cryptochromes.

To help resolve the apparently conflicting lines of reasoning and experimental evidence in the literature, we here present data from Arabidopsis, where sensitivity to applied MFs is mediated through a cryptochrome-dependent mechanism (Ahmad et al. 2007; Xu et al. 2012, 2014, 2015). Using two distinct and unrelated assays for cryptochrome biological activity, we probe the effects of a static MF of $500 \mu \mathrm{T}$ (10 $\times$ earth strength) on Arabidopsis placed under intermittent light/dark conditions. The MF was given solely during the dark interval between light pulses, such that there is a $10 \mathrm{~s}$ gap between the cessation of illumination and the onset of the MF. In this way, all transient radical pairs formed during forward electron transfer during the light period are eliminated from consideration as the possible magnetosensing step. Our results indicate that the plants, indeed, show response to the MF applied solely in the dark interval, consistent with data from the avian system (Wiltschko et al. 2016).

\section{Materials and methods}

\section{Plant materials and growth conditions}

Arabidopsis thaliana wild type (Wassilewskija) and phytochrome A, B deficient mutant lines (Ahmad and Cashmore 1997) were used for cry1 phosphorylation experiments. Transgenic cry 1 overexpressing lines as described (Ahmad et al. 2002) were used in hypocotyl growth tests. Seeds were sterilized by incubation with $25 \%$ bleach solution for $30 \mathrm{~min}$ and subsequently washed $3 \times$ with sterile water before plating. Seeds were plated on Petri dishes containing $2 \%(w / v)$ sucrose, 0.5X MS Murashige and Skoog Basal Salt Mixture pH 6.0 (MP biomedicals, INC, Illkirch, France), and $0.9 \%(\mathrm{w} / \mathrm{v})$ agar, and then maintained at $4{ }^{\circ} \mathrm{C}$ in the darkness for $48 \mathrm{~h}$. Petri plates were then illuminated with red light $(633 \mathrm{~nm})$ for $24 \mathrm{~h}$ to induce the germination, and then maintained in darkness for 4 days at $22{ }^{\circ} \mathrm{C}$ before the start of the seedling phosphorylation assay. For the seedling growth assay, seedlings were transferred from red light immediately to the test blue light and MF condition for 5 days growth.

\section{Magnetic field (MF) and blue-light exposure conditions}

All experiments were performed in an indoor controlledtemperature room $(10 \mathrm{~m} \times 2 \mathrm{~m} \times 2 \mathrm{~m}$ dimension $)$ at $23{ }^{\circ} \mathrm{C}$ with $46 \mu \mathrm{T}$ of local MF intensity. For the phosphorylation tests, we used a Helmholtz coil to generate a homogeneous $500 \mu \mathrm{T}$ static MF adjusted parallel to the local GMF. Each coil consisted of 20 windings of $1 \mathrm{~mm}$-diameter copper wire around a square plexiglass frame $(20 \times 20 \times 20 \mathrm{~cm}$ diameter) at a separation of $11.5 \mathrm{~cm}$ between coils. For the plant seedling growth experiments, two double wound matched Helmholtz coils as described in Ahmad et al. (2007) were used per experiment. The test coil generated a static MF of $500 \mu \mathrm{T}$ intensity at the plant seedling position. The control field condition was generated by cancelling the MF by currents running in opposite directions in the coils (see Ahmad et al. 2007 for more detailed description). The magnetic intensity in the cancelled field control coil conditions was $40 \mu \mathrm{T}$, which was the local GMF.

To provide blue-light exposure, $40 \mathrm{~mm}$-diameter round pre-mounted arrays of 7 high-intensity 'Royal Blue' (peak $448 \mathrm{~nm}$ ) blue-light LEDS (http://www.luxeonstar.com/ royal-blue-sinkpad-ii-40mm-7-uP-led-modules) were used together with a circular diffused optic array for optical diffusion of the beam. LEDs were placed $4.5 \mathrm{~cm}$ above the seedings at the center of the magnetic coils.

The activation of the magnetic coils and of the LEDs was controlled by custom-built automated programmable switches. These were created using a Raspberry Pi 3B. A 4-channel $5 \mathrm{~V}$ power relay board used GPIO pins on the Raspberry Pi to power on both the LED arrays and Helmholz coils. A Scratch program was written to control the GPIO pins connected to the power relay channels and enable the user to create the various light/magnetic field cycle patterns. In addition, a MAX31856 T-type thermocouple board was connected to GPIO pins on the Raspberry Pi and a T-type thermocouple was connected to this board to monitor temperature during the growth process. The MAX31856 board has an open-source python library that was leveraged to record the data from the thermocouple and save it to the raspberry throughout the course of the experiment in real time.

The programmable switch could, therefore, be set to control the current to the LEDs as well as to the MF coils to automatically and continuously generate the alternating 
pulses of blue light and MF exposure used in this study. Photon fluence of light intensity for the experiment was detected by a Quantum light meter (LI-185B, Li-Cor, Lincoln, NE, USA).

\section{Phosphorylation assay}

Arabidopsis phyAphyB double mutant etiolated seedlings that had been previously germinated in darkness for 4 days were exposed to the test condition (MF and/or blue-light exposure) as indicated in "Results". In cases, where the seedlings were exposed to MFs only in the dark interval between light pulses, an interval of $10 \mathrm{~s}$ after the end of the light period was inserted before the application of the MF. This resulted in the following programmed exposure condition: (1) $5 \mathrm{~min}$ blue light $\left(60 \mu \mathrm{mol} \mathrm{m}{ }^{-2} \mathrm{~s}^{-1}\right)$ at local GMF; (2) $10 \mathrm{~s}$ darkness at local GMF; (3) 9 min +50 s darkness at $500 \mu \mathrm{T}$ MF. This cycle was repeated six times in succession for this experiment. Subsequently, protein was extracted from plant seedlings and subjected to Western blotting. For the phosphorylation assay, we performed the analysis, as described in Shalitin et al. (2003). The intensity of the upper, phosphorylated band from the Western blotting [cry1(Pi)] was determined using the imaging software and expressed as a percentage of the intensity of the total cry1 protein (sum of phosphorylated plus unphosphorylated cry1) in the same lane. The formula is thereby $[$ cry $1(\mathrm{Pi})] /[$ cry 1 (total) $] \times 100$ yielding the percentage of phosphorylated cryptochrome per lane. Three triplicate lanes per individual experiment were averaged to yield the percent phosphorylation at the respective condition (MF or control). The percent phosphorylation between the MF and control conditions was then subtracted to yield the difference in phosphorylation between the test and control (MF vs. control) condition. Thus, a single percentage value of differential phosphorylation was obtained for each biological repeat (e.g., MF vs. control). In sum, five or six independent experiments were performed for each condition $( \pm \mathrm{MF})$, each involving three replicates.

To obtain the data plotted in Figs. 3, 4, 5, the percentage difference values (MF vs. control) originating from the five or six independent experiments were averaged. The values from these five or six independent experiments were then further subjected to statistical analysis, as described in "Materials and methods" to obtain the $P$ values, as plotted in Figs. 3, 4, 5.

\section{Western blotting}

All phosphorylation experiments were analyzed by Western blotting essentially as described (Ahmad et al. 2002; Xu et al. 2014). Triplicate samples of seedings were harvested from each plate per condition and homogenized in SDS-gel electrophoresis sample buffer (2\% SDS, 0.5 M Tris-HCl pH
6.8, 20\% glycerol, $100 \mathrm{mM} \beta$-mercaptoethanol, and $0.01 \%$ bromophenol blue), boiled for $10 \mathrm{~min}$. Gel electrophoresis was carried out in a 10\% SDS-PAGE separation gel: $30 \%$ bis/acrylamide stock (30\% stock solution from Sigma), $75 \mathrm{M}$ Tris $\mathrm{pH} 8.8,1 \%$ SDS, and resolved on a mini-gel apparatus (Amersham, Kingsport, TN, USA) for up to $4 \mathrm{~h}$ at $20 \mathrm{~mA}$. Resolved proteins were transferred to a nitrocellulose membrane (Amersham). The efficiency of protein transfer was verified by staining of blots with $2 \%$ ponceau $\mathrm{S}$ prior to detection by antibody. Anti-cry 1 antibody and detection procedure were as described previously (Ahmad et al. 2002; Bouly et al. 2007).

The detected cry1 antibody-specific bands were analyzed by the ImageJ $1.50 \mathrm{i}$ software $(\mathrm{NIH})$ to obtain digital values for signal intensities of the slower migrating (phosphorylated) and faster migrating (non-phosphorylated) bands as described previously (Shalitin et al. 2003; Xu et al. 2014). The extent of phosphorylation is expressed as the signal intensities of the slow-migrating bands normalized to the signal intensities of total cryptochrome protein (unphosphorylated plus phosphorylated cry1) in the same gel lane and represented numerically as a ration $(\operatorname{cry} 1(\mathrm{Pi}) / \mathrm{cry} 1)$. Each experimental sample plate was harvested in triplicate to obtain three separate readings per sample plate per experimental condition. The experimental value per condition was the mean of the three triplicate measurements. For each MF condition, duplicate plates were prepared, such that one plate was used for the test applied MF condition and the other maintained under the cancelled (geomagnetic) field condition, at the same position in the coil and identical illumination. The MF effect is expressed as a percentage of the response to the MF in the test condition in comparison with the response to the local (cancelled) field condition. Each such comparison experiment was repeated for six independent biological repeats performed on different days and using different batches of seeds.

To more accurately assess the effect of the MFs on CRY1 phosphorylation, and eliminate the possibility of unknown random artifact, we performed an additional control experiment as follows. We used an experiment model separated into 2 groups, the MF and control group (Fig. 1). For the MF group, we performed six independent biological repeats of a comparison between seedlings exposed to the experimental MF condition as compared to those that were not exposed to an applied field. This value provides the effect of the MF on the phosphorylation between the exposed group (+MF) and non-exposed group (-MF). For the Control group, we performed the identical six replicate biological repeats, but in this case, none of the two groups of samples were exposed to an applied MF. In this way, we obtained the comparison between 2 control untreated groups which were exposed only to the identical local geomagnetic field (GMF) condition $(40 \mu \mathrm{T})$. Since all plates were treated under the identical 


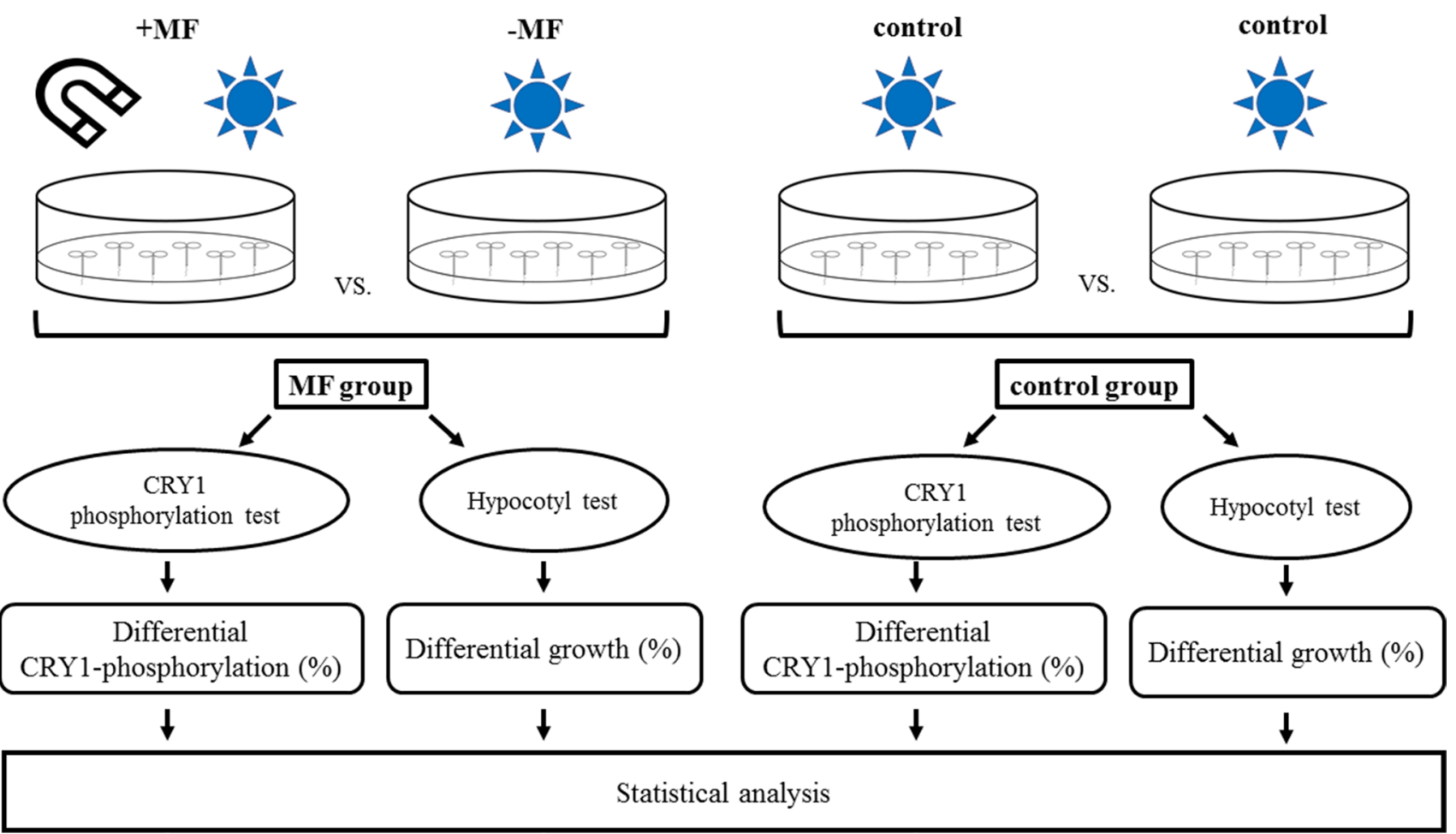

Fig. 1 Experimental design. For each test (hypocotyl growth or phosphorylation), two groups consisting of the experimental (MF) and the control group were evaluated. The MF group consisted of two matched experimental sub-groups for which we performed the comparison between $+\mathrm{MF}$ and $-\mathrm{MF}$ exposure condition. There were five or six independent biological replicates of all of the sub-groups. The control group also consisted of two matched experimental groups, divided into two sub-groups; however, in this case, none of the sam-

conditions, the control group would reveal any variability or artifact inherent in our experimental setup. Details of the control group and test group setup are described in Fig. 1.

\section{Hypocotyl growth experiment}

Subsequent to germination, cry1-overexpressing Arabidopsis seedlings were exposed to the following programmed test condition: (1) $5 \mathrm{~min}$ blue light $\left(60 \mu \mathrm{mol} \mathrm{m} \mathrm{m}^{-2}\right)$ at the local GMF; (2) $10 \mathrm{~s}$ darkness at local GMF; and (3) $9 \mathrm{~min}+50 \mathrm{~s}$ of darkness plus $500 \mu \mathrm{T}$ MF. This exposure regime was repeated continuously over a period of 5 days using the automated switch. Concomitantly with the test condition, in separate coil, a duplicate plate of seedlings was exposed to an identical light regime but with the MF cancelled by opposing currents running through the double wound coils (see Ahmad et al. 2007 for details of cancellation of the field). As a result, the seedlings in the cancelled field condition were never exposed to a $500 \mu \mathrm{T}$ MF. During the experiment, the temperature was monitored continuously and did not vary significantly between both coils. All experiments ples were exposed to magnetic field (MF), such that five or six replicate biological repeats were performed comparing the effect of the control condition on matched samples exposed to the identical control condition. This protocol detected the internal variation inherent in the experimental design. The results of each test were evaluated using the means from the five or six independent biological repeats. These were used to calculate average differential effect as well as to perform the statistical analysis

were performed double blind, such that the person measuring the seedlings did not know from under which condition they were taken. Hypocotyl lengths of 15 seedlings were measured per plate using the ImageJ 1.50i software (NIH). For each experiment, the mean seedling length in the test condition $(+\mathrm{MF})$ was expressed as a percentage of the mean seedling length of the local field (-MF) condition. Five independent biological repeats were performed under each condition to generate the average value presented in Fig. 3 for statistical analysis.

In addition to the comparison between seedlings with and without exposure to $500 \mu \mathrm{T}$ MF, we performed a control group experiment (Fig. 1), as for the phosphorylation experiments described above. For the control group, we performed the five replicate biological repeats, using two groups of plates grown under the same Helmholz coils as for the test hypocotyl growth experiments. However, in the control group experiments, none of the seedlings were exposed to an applied MF during the experiment (i.e., compared seedlings exposed to cancelled field to other seedlings exposed to cancelled field). In this way, we obtained the comparison 
between 2 control-treated groups which were in the identical local GMF condition $(40 \mu \mathrm{T})$. Since all plates were treated under the identical conditions, the control group would reveal any variability or artifact inherent in our experimental setup or due to inhomogeneous electromagnetic or otherwise conditions in the growth room, where the experiments were conducted.

\section{Data and statistical analysis}

As described above, all experiments involved five or six independent biological repeats comparing the effect of application of a $500 \mu \mathrm{T}$ MF to the identical untreated control seedlings. The MF effect was expressed as a differential percentage (treated vs. untreated sample) for each individual experiment in the MF group. In addition to the test experiments performed in this manner, a control group experiment was performed for each condition. In this case, all plates in a given experiment were treated with the identical light, but with the MF was cancelled. Therefore, no MF was applied to either test or control plates and the effect of the 'mock' magnetic field condition expressed as a differential percentage of the 'local field' condition (control vs. control sample), which were in fact identical. The data obtained from the MF group and control group were also subjected to the same analysis. The five or six independent values were then subject to statistical analysis (see below) and the average plotted on the graphs \pm SE. The schematic of this experimental design is shown in Fig. 1.

For statistical analysis, all data were analyzed by the SPSS Statistics program software IBM SPSS Statistics 23 (IBM). Data were analyzed for normality with the Shapiro-Wilk test $(P<0.05)$ and homogeneity of variances with Levene $(P<0.05)$. Results were expressed as mean \pm standard error of the mean (SE). The differences between the MF group and the control group were compared using Anova analysis. Differences were considered statistically significant with a $P$ value $<0.05(*),<0.01(* *)$, and $<0.001(* * *)$.

\section{Results}

There have been a number of reports implicating Arabidopsis cryptochromes in plant sensitivity to weak electromagnetic fields. These include effects of static MFs on plant deetiolation responses (Ahmad et al. 2007; Xu et al. 2012), on the onset of flowering (Xu et al. 2012, 2015), and on levels of cry-dependent gene expression and cryptochrome receptor phosphorylation (Xu et al. 2014). To obtain the most direct information on the effects of applied MFs on cryptochrome function, we have here replicated the previously reported cry1 phosphorylation assay which provides for an increased mobility shift on gels as a result of receptor activation (Xu et al. 2014). In this assay, the dark-adapted state of cryptochrome is unphosphorylated, as the C-terminal is folded to the protein surface. Upon illumination, the C-terminal of the protein unfolds, and multiple serine and threonine sites become exposed to phosphorylation by cellular kinases (Tan et al. 2013; Wang et al. 2015). In consequence, the protein undergoes a shift to a higher molecular weight which can be visualized in SDS protein gels (Fig. 2a). In sum, an upshift in protein molecular mass is a rapid, direct, and quantitative indicator of cryptochrome conformational change and biological activation in response to blue light.

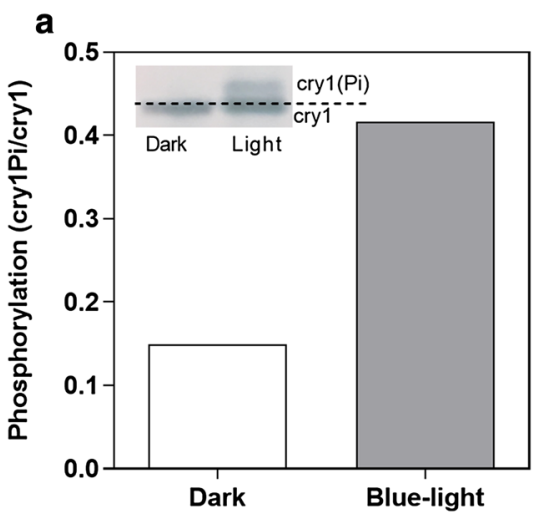

Fig. 2 Blue-light-dependent shift in Arabidopsis cry1 on Western blotting. a Wild-type seedlings (WS ecotype) were illuminated for $30 \mathrm{~min}$ at $60 \mu \mathrm{mol} \mathrm{m} \mathrm{s}^{-2} \mathrm{~s}^{-1}$ blue light intensity (light). This resulted in the appearance of an upper shifted phosphorylated band [inset - cry1(pi)] shown above the dotted line, consistent with the previous reports (Xu et al. 2014). Phosphorylation was quantitated as described in "Materials and methods", and expressed as a ratio of

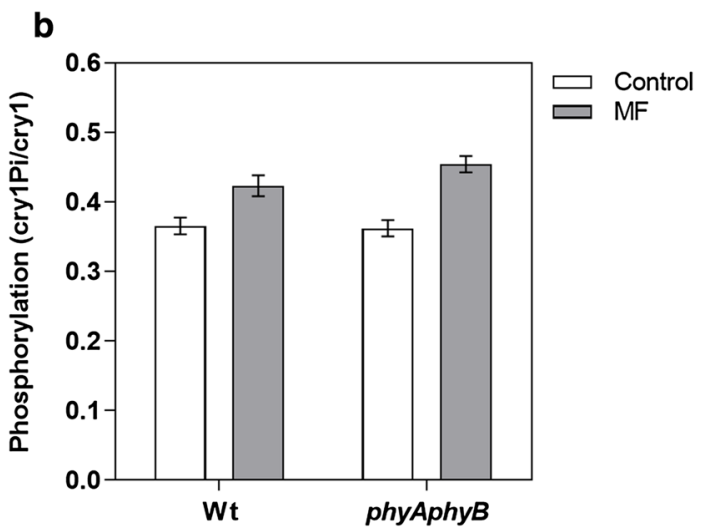

phosphorylated (upper shifted band) to total Cry1 protein. b Wild type and phyAphyB mutant Arabidopsis seedlings were illuminated for $30 \mathrm{~min}$ at $60 \mu \mathrm{mol} \mathrm{m} \mathrm{m}^{-2} \mathrm{~s}^{-1}$ blue light intensity, in either the absence (control) or presence (MF) of a $500 \mu \mathrm{T}$ static magnetic field. Error bar represents \pm SE of measurements from three independently analyzed seedling samples per condition 


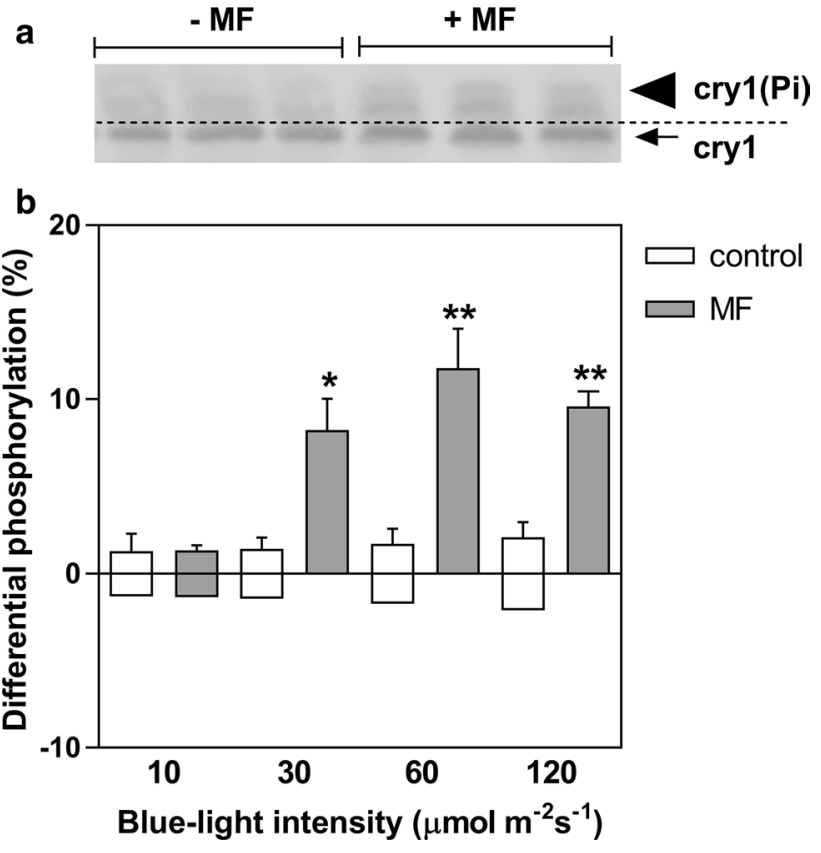

Fig. 3 Blue light intensity-dependent phosphorylation of cry1 in $500 \mu \mathrm{T}$ static magnetic field. a Immunoblots of samples from a single experiment. Each lane represents homogenates of independently harvested phyAphyB Arabidopsis seedling sample exposed to blue light for $30 \mathrm{~min}$ at a fluence rate $60 \mu \mathrm{mol} \mathrm{m} \mathrm{m}^{-2} \mathrm{~s}^{-1}$ and to $500 \mu \mathrm{T}$ of magnetic field $(+\mathrm{MF})$ or else to the geomagnetic field $(-\mathrm{MF})$. This provided triplicate readings under the same illumination conditions for each experimental determination in a single experiment. The slower migrating phosphorylated cry $1(\mathrm{Pi})$ are indicated by the arrowhead. The level of cry1 phosphorylation was represented as the ratio [cry1(Pi)/cry1] for each lane. The value from the triplicate measurements was averaged to determine the phosphorylation value in each experiment. b PhyAphyB seedlings were irradiated at the indicated blue light intensity over $30 \mathrm{~min}$ illumination time. For the test (MF) experiments, the percentage difference in cry1 phosphorylation between samples exposed to the magnetic field and samples exposed only to the local field were averaged from six independent experiments (see Fig. 2a for determination of a single experimental data point). For the control experiments, the identical procedure was followed except that the samples were exposed to a 'mock' magnetic field identical to the local field (see "Materials and methods"). Data represent the means and \pm SE of six independent experiments. Significance levels: $P$ value $<0.05(*)$ and $P$ value $<0.01(* *)$

In the prior study, it had been reported that exposure of etiolated Arabidopsis seedlings to a static MF of $500 \mu \mathrm{T}$ resulted in a significant increase in phosphorylation of Cry1 relative to control seedlings (Xu et al. 2014). We, therefore, began replicating the conditions of this prior study using wild-type seedlings of ecotype Wassilewskija. In our experiments, we illuminated the wild-type seedlings at $60 \mu \mathrm{mol} \mathrm{m} \mathrm{m}^{-2} \mathrm{~s}^{-1}$ blue light for $30 \mathrm{~min}$ in the absence (control) or the presence (MF) of a $500 \mu \mathrm{T}$ applied staticMF, which is roughly tenfold the earth's GMF. In our initial experiment, we obtained a measurable increase in a
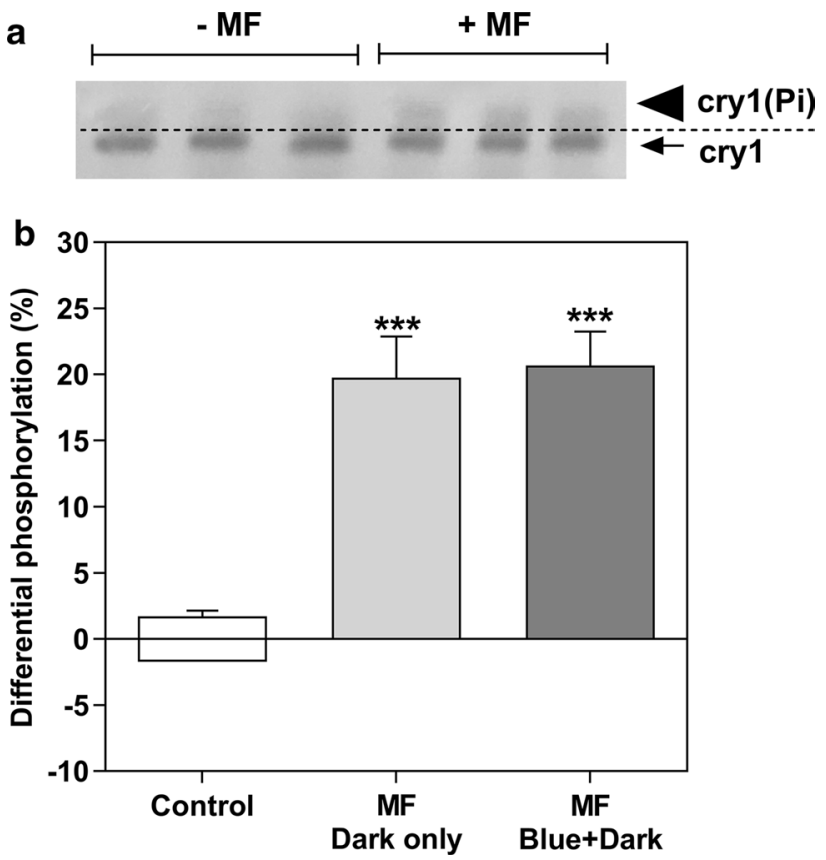

Fig. 4 Phosphorylation of cry1 in $500 \mu \mathrm{T}$ magnetic field under light/ dark cycles. a Immunoblots of a representative experiment of phyAphy $B$ seedlings exposed to blue light/dark cycle (blue light at a fluence rate $60 \mu \mathrm{mol} \mathrm{m} \mathrm{m}^{-2} \mathrm{~s}^{-1}$ for $5 \mathrm{~min}$ and followed by $10 \mathrm{~min}$ in dark, cycled for $90 \mathrm{~min})$. The $500 \mu \mathrm{T}$ magnetic field was applied only in the dark interval (+MF), compared to the sample in geomagnetic field ($\mathrm{MF}$ ). The signal intensity of the phosphorylated cry1(Pi) (indicated by the arrowhead) is normalized to the total cry 1 protein and represented as a ratio [cry1(Pi)/cry1] for each lane. The average value of the three triplicate measurements is taken as the phosphorylation value for one experimental condition. Each experiment was repeated at least five times to obtain the average values plotted in the lower part of figure. b PhyAphyB seedlings were illuminated under light pulse conditions as in Fig. 3a. A $500 \mu \mathrm{T}$ static magnetic field was applied either in the dark interval (MF dark only) or throughout the entire light and dark intervals (MF blue + dark). Control experiments represented mock-treated seedlings (no magnetic field applied under either test or control condition-see "Materials and methods"). The percentage values represent the percentage difference in phosphorylation in seedlings resulting from exposure to the MF under the indicated illumination conditions. Effect of magnetic field occurs even if the field is applied solely in the dark interval between light pulses. Data are means and $\pm \mathrm{SE}$ of six independent experiments). $* * *$ Significance levels from statistical analysis: $P<0.001$

phosphorylation due to application of the MF (Fig. 2b), consistent with the prior report (Xu et al. 2014).

We next attempted to improve the sensitivity of the assay, by testing the effects of applied MFs on seedlings that are deficient in the red/far red receptor phytochrome. Phytochrome is known to interact with cryptochrome at many levels and enhances and maintains plant downstream responses to blue light (Ahmad and Cashmore 1997; Mazzella et al. 2001). These interactions, furthermore, include a direct phosphorylation of the C-terminal domain of cryptochrome by phytochrome A, which likely also occurs 
a
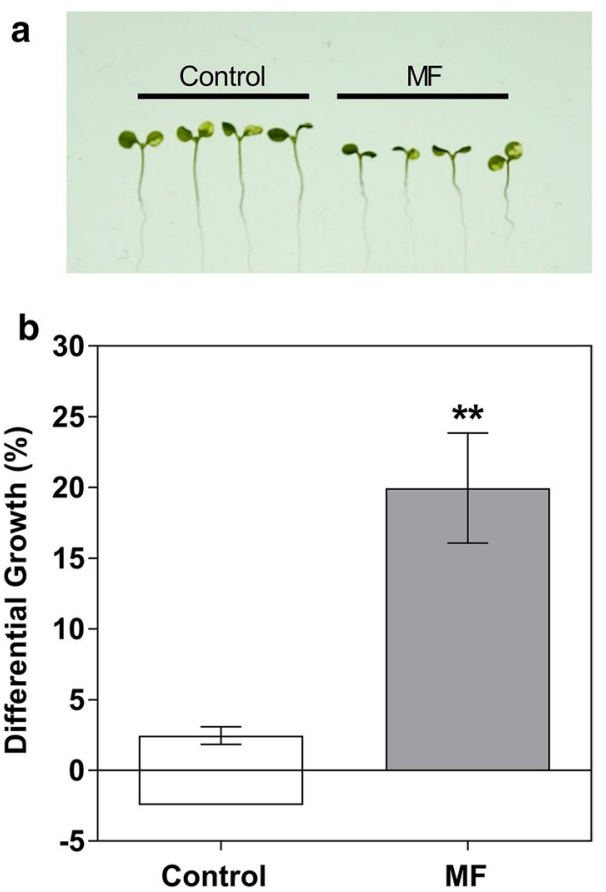

Fig. 5 Effect of $500 \mu \mathrm{T}$ on Arabidopsis seedling hypocotyl growth inhibition (a). Seedlings were exposed for 5 days to repeated cycles of pulsed blue light ( 5 min light followed by 10 min darkness) in the presence or absence of an applied static magnetic field. b Differential growth of seedling exposed to the magnetic field as compared to the control (local field) seedlings is presented (MF group). For the control group, all seedlings in the experiments were exposed only to local field. Data are mean and \pm SE of five independent experiment. ${ }^{*}$ Significance levels for $t$ test: $P<0.01$

in vivo (Ahmad et al. 1998). Therefore, phytochrome action could mask and reduce any visible effect of an externally applied MF on cryptochrome responses, as phytochromes do not form radical pairs required for chemical magnetosensing. Indeed, we found that the phyAphyB deficient mutants showed a visibly enhanced response to an applied MF in our preliminary experiments (Fig. 2b). For all subsequent phosphorylation experiments, we, therefore, used the phyAphy mutant seedling lines.

Cryptochrome undergoes a photocycle, whereby flavin is reduced in response to light. The extent of flavin reduction is determined by the light intensity at quantum efficiencies that have been calculated empirically from in vitro and in vivo experiments (Procopio et al. 2016). Therefore, for magnetic sensitivity to occur, the light intensity should be at an intensity that is below saturation, but sufficiently above threshold for small changes in cryptochrome sensitivity to be readily visible in the phenotypic response. To assess the optimum illumination conditions for observing magnetic sensitivity, we accordingly performed phosphorylation experiments at increasing blue-light intensities ranging from 10 to $120 \mu \mathrm{mol} \mathrm{m} \mathrm{m}^{-2} \mathrm{~s}^{-1}$. Phosphorylation under the applied
MF condition was expressed as a percentage of the phosphorylation obtained under identical illumination conditions in the absence of the applied field. Six duplicate experiments were performed for each condition, involving independent biological repeats (see "Materials and methods"). Temperature was measured by a thermocouple attached to a computer and monitored throughout the experiment.

The MF sensitivity was expressed as the percent increase in phosphorylation in the presence of an MF as compared to that of seedlings in the control condition. Statistical analysis was performed on six independently performed experiments to obtain statistical $P$ values (see "Materials and methods").

There are many hidden variables that may affect perception of MFs in plants and other organisms, which has made such studies historically difficult to replicate. For example, the existence of high concentrations of metal in the building, or of random electromagnetic noise or of electronic background signals can apparently perturb avian directional sensing which is presumed to follow from a cryptochromedependent mechanism (see, e.g., Engels et al. 2014). We, therefore, devised an additional control experiment to eliminate all possible artifacts due to the experimental setup or the environment in the room, where the experiments were performed. In this additional 'control' experiment, we performed six independent biological repeats of the illumination treatments exactly as for the MF test experiment. However, in the control experiment, none of the plates had received an applied MF, and therefore, in this experiment, we measured the percentage phosphorylation differences between each of six 'mock'-treated plates to each of six untreated plates. In this way, the inherent variation in our experimental setup could be unequivocally determined. Further details of the experimental design and procedures, image analysis, and statistical treatments are found in "Materials and methods".

The results of this analysis are shown in Fig. 3. The unphosphorylated cry1 (lower band) and phosphorylated cry 1pi (upper band) from triplicate seedling samples from a single experiment at $60 \mu \mathrm{mol} \mathrm{m}{ }^{-2} \mathrm{~s}^{-1}$ blue light for $30 \mathrm{~min}$ are indicated by arrows (Fig. 3a). Quantitation of the phosphorylation was performed by the imaging software to determine the ratio of phosphorylated (upper band) to unphosphorylated (lower band) cry 1 protein in each lane; then, the three independently determined values were averaged to obtain a numerical value for the phosphorylation under this condition. The effect of the MF for this single experiment was then expressed as the percentage change in phosphorylation between the MF exposed (average of 3 sample readings) as compared to the unexposed or 'mock exposed' samples (also averaged from 3 sample readings). The experiments were subsequently repeated in full at least five times.

The data presented in Fig. $3 \mathrm{~b}$ represent the average of all of the six independent biological repeats, comparing the increase in phosphorylation after exposure to a $500 \mu \mathrm{T}$ MF 
(MF, grey squares) at the indicated blue-light intensities. For the control experiments (control, white squares), six independent experiments were performed comparing phosphorylation in non-exposed seedlings to 'mock' exposed seedlings, such that neither sample group had been exposed to an MF (see "Materials and methods").

As expected, there was no significant variation in phosphorylation in the control samples, which consisted of repetitions under identical conditions of 6 independent biological repeats of pairs of samples receiving the same exposure to the local MF. The control sample thereby represents the inherent variation of the experimental method used for each condition. By contrast, under 30, 60, and $120 \mu \mathrm{mol} \mathrm{m} \mathrm{m}^{-2}$ $\mathrm{s}^{-1}$ blue-light illumination, there was a significant increase in phosphorylation as a result of exposure to the MF. The maximal effect was about $12 \%$ increased phosphorylation in seedlings exposed to the MF as compared to control seedlings $(P$ value $<0.01)$ at $60 \mu \mathrm{mol} \mathrm{m} \mathrm{m}^{-2} \mathrm{~s}^{-1}$, which occurs well below saturation in fluence response range deduced for Cry1 (Procopio et al. 2016).

To date, two distinct steps in the cryptochrome redox cycle have come under consideration as possible points of interaction with an MF. The first occurs during forward (light-driven) electron transfer, where electrons are transferred from the protein surface to the flavin via a chain of intraprotein electron transfer involving $\operatorname{Trp}^{\circ}$ and $\operatorname{Tyr}^{\circ}$ radicals. In this case, the radical pairs are short-lived intermediates with a maximum lifetime of less than $100 \mathrm{~ms}$ (Thöing et al. 2015; Nohr et al. 2016) in the isolated proteins. By contrast, evidence from the avian system has shown that magnetic sensitivity can occur in darkness if MF pulses are given in the dark periods between pulses of illumination (Wiltschko et al. 2016). In this way, short-lived radical pairs such as formed during forward electron transfer are eliminated from consideration as possible targets for interaction with MFs, as they decay too rapidly to be present when the $\mathrm{MF}$ is applied in the dark intervals.

To resolve the question of what step of the plant cryptochrome photocycle forms the radical pairs necessary for magnetic sensitivity, we designed a series of light/dark pulse experiments analogous to the avian experiments. In the case of plant cryptochrome, light-induced phosphorylation requires several min to become measureable (Shalitin et al. 2003), and the cryptochrome signal is also seen to persist for several min in complete darkness after the end of the light pulse (Bouly et al. 2007; Herbel et al. 2013). We, therefore, pulsed blue light at fluence rate of $60 \mu \mathrm{mol} \mathrm{m} \mathrm{m}^{-2} \mathrm{~s}^{-1}$ for $5 \mathrm{~min}$, followed by a $10 \mathrm{~min}$ dark interval, and repeated this light/dark cycle until the total time amounted to $90 \mathrm{~min}$ (three complete light/dark cycles) (Fig. 4). We first established whether cryptochrome-dependent magnetic sensitivity can be observed under this light regime by assessing cryptochrome phosphorylation in seedlings exposed to a
$500 \mu \mathrm{T}$ MF. Under the alternating light/dark illumination conditions, there was a $20 \%$ increase in cryptochrome phosphorylation in seedlings exposed to the MF relative to the control (non-exposed) seedlings ( $P$ value $<0.001)$ (Fig. 4). The appropriate control experiment, where samples that had not been exposed to the MF were compared to 'mock' exposed samples under the identical illumination conditions (see "Materials and methods"), showed no such change in phosphorylation indicating that the experimental setup does not introduce artifact.

Next, we determined whether the response to the MF could only occur when the MF was applied at the same time as the illumination. For this experiments, we use the $5 \mathrm{~min}$ blue light/10 min dark repeated pulsed light condition as described above; however, we applied the static field only during the 10 min dark intervals between the 5 min light pulses. To ensure that no radicals formed under conditions of illumination were carried over into the subsequent dark period, we provided a $10 \mathrm{~s}$ gap in darkness after the end of the illumination period and the onset of the MF. The results showed that there was a $19 \%$ increase in phosphorylation of cry 1 under this regime ( $P$ value $<0.001$; Fig. 4$)$. These results show that the MF effect on cryptochrome occurs during the process of the return of the receptor to its resting state, corresponding to the period of flavin reoxidation in the cryptochorme photocycle (Müller and Ahmad 2011).

As an additional and complementary test for cryptochrome response to MFs, we used the hypocotyl growth inhibition assay, which is a cryptochrome-dependent response under blue light which has been previously shown to be modulated by an applied MF (Ahmad et al. 2007). We have here modified and optimized this assay as compared to prior studies (Ahmad et al. 2007) using transgenic seedlings which overexpress the Cry1 receptor protein. In these lines, cryptochrome protein expression is enhanced five times over background levels leading to correspondingly enhanced cryptochrome function (Ahmad et al. 2002). In addition, we have conducted the growth experiments over 5 days instead of $72 \mathrm{~h}$ as in prior studies, and used the illumination conditions which showed the optimum response to the applied MF for the phosphorylation experiments (from Fig. 4).

Transgenic cry 1 overexpressing seedlings were germinated as described in "Materials and methods" and then placed under continuous alternating light/dark pulses of $5 \mathrm{~min}$ at $60 \mu \mathrm{mol} \mathrm{m} \mathrm{m}^{-2} \mathrm{~s}^{-1}$ blue light followed by $10 \mathrm{~min}$ darkness, over a period of 5 full days. Matched plates were placed under each of two Helmholtz coils as described (Ahmad et al. 2007). One coil was programmed to provide a $500 \mu \mathrm{T}$ static MF during the dark interval between light pulses, with in addition, a $10 \mathrm{~s}$ dark interval between the cessation of the light and the onset of the MF. The second coil was programmed to provide a cancelled MF (current running in opposite directions in the double wound coil, such that the 
MF remained at local (geomagnetic) field strength, although the current was identical to the test coil). After the end of the experimental period, the hypocotyl length of 15 seedlings from the MF-treated plates was averaged per condition. As can be seen (Fig. 5a), application of the MF resulted in substantially reduced hypocotyl growth, indicative of enhanced cryptochrome function. This result is in agreement with the phosphorylation experiments (Figs. 3, 4) and with prior studies (Ahmad et al. 2007; Xu et al. 2014). The results presented in Fig. $5 \mathrm{~b}$ represent the average percentage of differential growth obtained from five independent plant growth experiments, with exposure to the MF only during the dark interval. Statistical analysis showed a differential growth of $20 \%$, at $P$ value $<0.01$, in good agreement with results from the phosphorylation experiments (Fig. 4).

To eliminate the possibility of artifact ensued from unknown electromagnetic background in the test chamber, and to get a baseline value for the inherent variability in our experimental setup, we again performed the entire experiment using the identical magnetic coils and illumination conditions, in the absence of an MF. That is, both plates of a matched set of seedlings were grown under identical illumination conditions, at the identical positions in the Helmholz coils as the MF test experiments, and both receiving the identical mock treatments (antiparallel cancelled fields). In other words, all seedlings in the test experiment received the same, earth strength MF. As expected, the results of this control experiment showed a variability of only $\pm 2.5 \%$, with no statistical significance in seedling length between the two groups of seedlings under the two coils.

Therefore, the effect of the applied MF under the test condition is conclusive and provides evidence that the effect of the static MF on the cryptochrome receptor occurs at a step on the reaction pathway that is independent of light.

\section{Discussion}

The suggestion that a biological response to weak (near earth strength) MFs can be mediated by a radical-pair (RP) mechanism was first proposed by Schulten et al. (1976) and has gained wide spread acceptance in the past 30 years. In particular, a broad literature has shown experimentally that the rate, yield, and/or product distribution of chemical reactions which involve radical intermediates can, indeed, be modified by MFs (Rodgers 2009). For biological responses, cryptochrome receptors had been initially proposed as possible magnetosensors by Ritz et al. (2000) and subsequently implicated experimentally in MF sensitivity in organisms ranging from plants to fruit flies and migratory birds (Ritz et al. 2010; Hore and Mouritsen 2016). The current challenge is to determine the underlying chemistry of how the MF can interact with this biological sensor, and in particular to identify the relevant radical-pair intermediates and reaction rates that are altered.

Up until now, attention has been focussed primarily on the forward electron transfer reaction of cryptochromes, whereby excited state flavin is reduced through a chain of intramolecular electron transfer involving $\operatorname{Trp}^{\circ}$ and $\operatorname{Tyr}^{\circ} \mathrm{rad}-$ icals. The resulting $\operatorname{Trp}^{\circ} / \mathrm{FADH}^{\circ}$ or $\operatorname{Trp}^{\circ} / \mathrm{FAD}^{\circ}{ }^{\circ}$ radicals are presumed targets for electromagnetic fields, which as a consequence alter the rate of flavin photoreduction which leads to biological activation (see Ahmad 2016; Ganguly et al. 2016; Lin et al. 2018) for current discussion of the role of redox state interconversion in cryptochrome photo-sensing). This reaction has been deemed particularly suitable for magnetic sensitivity on theoretical grounds (see, e.g., Mouritsen and Hore 2012; Dodson et al. 2013; Lüdemann et al. 2015; Hong and Pachter 2015; Worster et al. 2016). It has also been shown experimentally that applied MFs can alter flavin reduction rates using isolated cryptochrome proteins from plants and Drosophila, albeit at high (mT) MF intensities (Maeda et al. 2012; Sheppard et al. 2017). However, recent evidence from the avian system has shown that the Trp $\%$ $\mathrm{FADH}^{\circ}$ radical pair cannot be involved in magnetosensing, as the birds are oriented in green light during which these radicals are not formed (Nießner et al. 2013). Furthermore, it has been shown that birds subjected to alternating pulses of light and darkness show directional magnetic sensitivity even when the MF is applied solely during the dark intervals between light pulses (Wiltschko et al. 2016). These results are inconsistent with forward electron transfer to the flavin as the reaction step at which the MF can act.

In this study, we have examined the magnetic sensitivity of Arabidopsis cryptochrome in light of the known parameters of the cryptochrome photocycle (Procopio et al. 2016). To ensure reproducibility of our methods, we have replicated a reported direct assay from another lab for MF sensitivity of cryptochrome photoreceptor phosphorylation (Xu et al. 2014). This is a rapid assay (30-90 min duration) that measures changes in conformational state at the level of the photoreceptor molecule itself. It results from C-terminal conformational changes which occur upon cryptochrome activation and which render phosphorylation sites accessible to cellular kinases (Tan et al. 2013; Wang et al. 2015). Phosphorylation only occurs in response to blue light, as it is a direct consequence of cryptochrome photoreceptor activation (Tan et al. 2013; Wang et al. 2015). However, continuous light is not necessary for biological activity as the cryptochrome active (photoreduced) redox state is stable for a period of several min even after return of the system to darkness (Bouly et al. 2007; Herbel et al. 2013; Procopio et al. 2016). Therefore, robust cryptochrome activation, which includes phosphorylation, can be observed under pulsed light conditions, wherein brief successive periods of illumination are alternated with periods of complete darkness. 
Under continuous illumination, using the phosphorylation assay, we observed an increase in cryptochrome phosphorylation in response to an applied MF relative to control seedlings maintained under the same illumination conditions but without applied field. This indicated that the applied $500 \mu \mathrm{T}$ field enhanced the cryptochrome biological response (extent of receptor activation) to illumination, consistent with reports from the prior study using this method (Xu et al. 2014). Using pulsed light conditions of 5 min light alternating with 10 min of dark, we also observed cryptochrome sensitivity to an applied MF, in this case even more pronounced than under continuous illumination. Significantly, applying the MF solely in the dark intervals between bluelight illumination pulses resulted in the same stimulation of protein phosphorylation as found when the MF was applied during both light and dark intervals (continuously). This indicates that the MF cannot act at a reaction step in the cryptochrome photocycle that involves the forward electron transfer pathway, as these radical-pair intermediates (Trp\% $\mathrm{FADH}^{\circ}$ or $\mathrm{Tyr}^{\circ} / \mathrm{FADH}^{\circ}$ ) have reported lifetimes only in the millisecond time scale (see, e.g., Thöing et al. 2015).

These results were further confirmed by use of an independent assay for cryptochrome photoreceptor function, namely, inhibition of hypocotyl elongation in Arabidopsis seedlings. In this assay, seedling growth is inhibited in response to blue light through activation of cryptochrome, such that the greater the cryptochrome biological activity, the shorter the seedling hypocotyl length in response to blue light. Application of weak MFs has been reported to alter hypocotyl growth inhibition in the past studies (Ahmad et al. 2007; Xu et al. 2012), although other labs have reported difficulty in reproducing these results (Harris et al. 2009).

We believe that the reasons for variable results among different labs attempting the hypocotyl growth inhibition protocol are due to the already weak nature of the reported biological response and the many cellular factors (including the action of hormones, phytochromes, and other metabolic processes) that contribute to elongation growth and thereby mask the effect of cryptochromes (see, e.g., Cheng et al. 2013; Hu et al. 2017). Furthermore, different lab environments may have differing amounts of metal or electronic equipment generating background electromagnetic noise (Engels et al. 2014), and also there could be other forms of interference with the relatively weak MFs used to elicit differential plant growth responses. Therefore, to ensure reproducibility of this method under variable growth conditions in different labs, we have optimized the hypocotyl growth assay in the current study using cryptochrome overexpressing seedlings. These seedlings have approximately fivefold increase in cryptochrome with respect to wild-type seedlings, such that the cryptochrome-dependent signal effectively dominates the growth inhibition response in the expressing plant and reduces the interference from additional growth factors (Ahmad et al. 2002). We have also further increased the period of seedling growth from 3 to 5 days, to maximize the effect of differential growth under the applied MF, and used the optimized illumination protocols developed in the current study. Using this optimized hypocotyl growth inhibition protocol, we observed approximately $20 \%$ reduced seedling growth upon application of an applied MF (Fig. 5). Moreover, this response was obtained even when the MF was applied exclusively in the dark interval between successive light pulses (Fig. 5).

In sum, using two independent and unrelated assays for cryptochrome biological activation, we have shown that application of a weak MF of $500 \mu \mathrm{T}$ results in an enhanced cryptochrome response. We have further shown that a response occurs even when the MF is applied solely in the dark interval between blue-light pulses. Significantly, in these pulsed light experiments, the MF was applied a full $10 \mathrm{~s}$ after the cessation of the preceding light period. From these results, it can be concluded that no short-lived transient reaction intermediate formed during forward electron transfer (flavin photoreduction) can possibly be the target for an MF effect mediated by the plant cryptochrome. Rather, the radical pair impacted by the MF must be formed during an extended period consistent with reoxidation of the flavin, which is a light-independent process requiring molecular oxygen (Müller and Ahmad 2011) and that involves the formation of reactive oxygen species (ROS) and/or superoxide radicals. By altering the rate constant for flavin reoxidation, the MF could change the ratio of the different redox states of cryptochrome and thereby alter its biological activity (see Fig. 6 for model of the effect of the MF on the cryptochrome photocycle). It should be added that attempts in the literature to link biological magnetosensing to forward electron transfer events in cryptochrome (see, e.g., Solov'yov et al. 2007; Maeda et al. 2012; Mouritsen and Hore 2012; Dodson et al. 2013; Biskup 2013; Evans et al. 2015; Hong and Pachter 2015; Lüdemann et al. 2015; Müller et al. 2015; Bialas et al. 2016; Kattnig et al. 2016; Nohr et al. 2016, 2017; Worster et al. 2016; Van der Straeten et al. 2018) are not supported by the biological data neither in birds (Wiltschko et al. 2016) nor (as shown in this study) in plants.

At present, theoretical considerations have argued against a flavin/superoxide radical pair, which is formed in the course of flavin reoxidation (Müller and Ahmad 2011), as the magnetosensing intermediate in cryptochromes (reviewed in Hore and Mouritsen 2016). However, cryptochrome localized within living cells is in contact with many cellular metabolites, which, moreover, can move into the flavin pocket in close association with the flavin cofactor (see, e.g., Engelhard et al. 2014). Therefore, the possibility of the third party cellular factors participating in the formation of radical pairs during the process of cryptochrome flavin reoxidation cannot be excluded. Finally, there is as yet no 


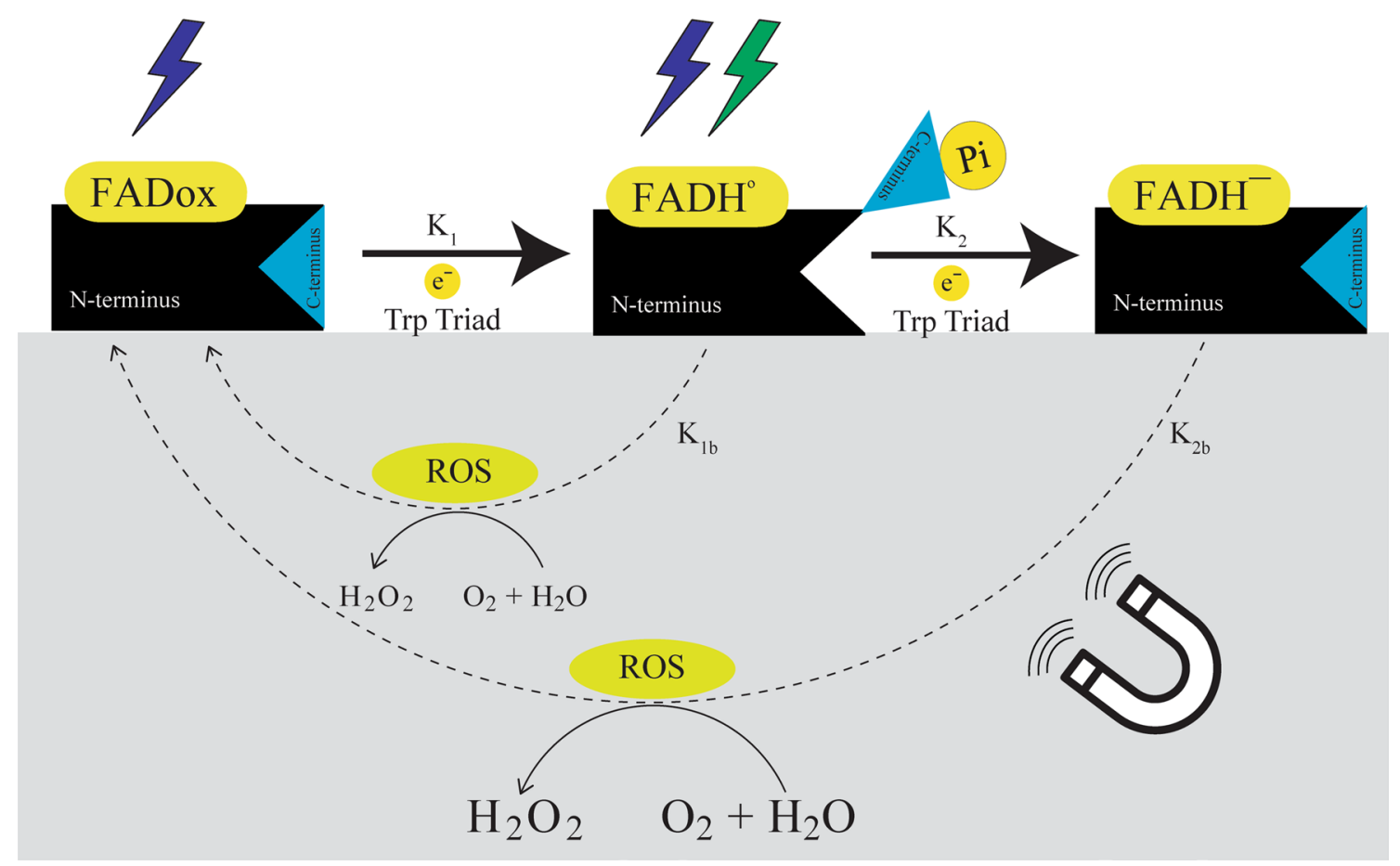

Fig. 6 Model of magnetic field effect on the cryptochrome photocycle. Cryptochrome is activated by flavin reduction which triggers conformational change leading to unfolding and subsequent phosphorylation of the C-terminal domain. The flavin is subsequently reoxidized by reaction with molecular oxygen that occurs independently of light

conclusive evidence that cryptochrome is, in fact, a magnetosensor. Indeed, there have been reports of magnetosensing, whereby cryptochromes interact with an iron-based magnetosensor MagR (Qin et al. 2016) as opposed to the current radical-pair-based (chemical) mechanism (Hore and Mouritsen 2016) discussed here. Future theoretical studies taking all of these possibilities into account, such as properties of potential scavenging intermediates in magnetic sensitivity (Kattnig and Hore 2017), are, therefore, required to provide the necessary framework for further experimental work to identify the actual magnetosensing intermediate states.

Author contribution MA designed the experiments, MA, CFM, JL, and MP wrote the paper; MP and LDA conducted experiments, DB, CFM, JL built equipment and designed analytical tools, MA and MP analyzed the data, and all authors read and approved the manuscript.

Acknowledgements We are indebted to Adam Purvis, Dorothy Engle and Haider Raad for valuable technical assistance and support at many stages of the project. Support was from NSF (\#1658640), AFOSR (FA9550-14-0-0409) and AFOSR (FA9550-17-1-0458).

Open Access This article is distributed under the terms of the Creative Commons Attribution 4.0 International License (http://creativeco
(Ahmad 2016). The effect of an applied magnetic field on the cryptochrome photocycle occurs during the period of flavin reoxidation. The most likely effect is to alter the rate constant of reoxidation of the reduced flavin intermediates, and thereby alter the lifetime of the activated state

mmons.org/licenses/by/4.0/), which permits unrestricted use, distribution, and reproduction in any medium, provided you give appropriate credit to the original author(s) and the source, provide a link to the Creative Commons license, and indicate if changes were made.

\section{References}

Ahmad M (2016) Photocycle and signaling mechanisms of plant cryptochromes. Curr Opin Plant Biol 33:88-105. https://doi. org/10.1016/j.pbi.2016.06.013

Ahmad M, Cashmore AR (1997) The blue-light receptor cryptochrome 1 shows functional dependence on phytochrome A or phytochrome B in Arabidopsis thaliana. Plant J 11:421-427. https ://doi.org/10.1046/j.1365-313X.1997.11030421.x

Ahmad M, Jarillo JA, Smirnova O, Cashmore AR (1998) The CRY1 blue light photoreceptor of Arabidopsis interacts with phytochrome A in vitro. Mol Cell 1(7):939-948

Ahmad M, Grancher N, Heil M, Black RC, Giovani B, Galland P, Lardemer D (2002) Action spectrum for cryptochrome-dependent hypocotyl growth inhibition in Arabidopsis. Plant Physiol 129(2):774-785

Ahmad M, Galland P, Ritz T, Wiltschko R, Wiltschko W (2007) Magnetic intensity affects cryptochrome-dependent responses in Arabidopsis thaliana. Planta 225(3):615-624. https://doi. org/10.1007/s00425-006-0383-0

Banerjee R, Schleicher E, Meier S, Viana RM, Pokorny R, Ahmad M, Bittl R, Batschauer A (2007) The signaling state of Arabidopsis cryptochrome 2 contains flavin semiquinone. J Biol Chem 282:14916-14922. https://doi.org/10.1074/jbc.M700616200 
Bialas C, Jarocha LE, Henbest KB, Zollitsch TM, Kodali G, Timmel CR, Mackenzie SR, Dutton PL, Moser CC, Hore PJ (2016) Engineering an artificial flavoprotein magnetosensor. J Am Chem Soc 138(51):16584-16587. https://doi.org/10.1021/jacs.6b09682

Biskup T (2013) Time-resolved electron paramagnetic resonance of radical pair intermediates in cryptochromes. Mol Phys 111(24):3698-3703. https://doi.org/10.1080/00268 976.2013.833350

Bouly JP, Schleicher E, Dionisio-Sese M, Vandenbussche F, Van Der Straeten D, Bakrim N, Meier S, Batschauer A, Galland P, Bittl R, Ahmad M (2007) Cryptochrome blue light photoreceptors are activated through interconversion of flavin redox states. J Biol Chem 282:9383-9391. https://doi.org/10.1074/jbc.M609842200

Chaves I, Pokorny R, Byrdin M, Hoang N, Ritz T, Brettel K, Essen L-OO, van der Horst GT, Batschauer A, Ahmad M (2011) The cryptochromes: blue light photoreceptors in plants and animals. Annu Rev Plant Biol 62:335-364. https://doi.org/10.1146/annur ev-arplant-042110-103759

Cheng X, Ruyter-Spira C, Bouwmeester H (2013) The interaction between strigolactones and other plant hormones in the regulation of plant development. Front Plant Sci 4:199. https://doi. org/10.3389/fpls.2013.00199

Dodson CA, Hore PJ, Wallace MI (2013) A radical sense of direction: signaling and mechanism in cryptochrome magnetoreception. Trends Biochem Sci 38:435-446

Engelhard C, Wang X, Robles D, Moldt J, Essen LO, Batschauer A, Bittl R, Ahmad M (2014) Cellular metabolites enhance the light sensitivity of Arabidopsis cryptochrome through alternate electron transfer pathways. Plant Cell 26(11):4519-31.https://doi. org/10.1105/tpc.114.129809

Engels S, Schneider NL, Lefeldt N, Hein CM, Zapka M, Michalik A, Elbers D, Kittel A, Hore PJ, Mouritsen H (2014) Anthropogenic electromagnetic noise disrupts magnetic compass orientation in a migratory bird. Nature 509:353-356. https://doi.org/10.1038/ nature 13290

Evans EW, Li J, Storey JG, Maeda K, Henbest KB, Dodson CA, Hore PJ, Mackenzie SR, Timmel CR (2015) Sensitive fluorescencebased detection of magnetic field effects in photoreactions of flavins. Phys Chem Chem Phys 17:18456-18463. https://doi. org/10.1039/c5cp00723b

Fedele G, Green EW, Rosato E, Kyriacou CP (2014) An electromagnetic field disrupts negative geotaxis in Drosophila via a CRYdependent pathway. Nat Commun 5:4391. https://doi.org/10.1038/ ncomms5391

Foley LE, Gegear RJ, Reppert SM (2011) Human cryptochrome exhibits light-dependent magnetosensitivity. Nat Commun 2:356. https ://doi.org/10.1038/ncomms1364

Ganguly A, Manahan CC, Top D, Yee EF, Lin C, Young MW, Thiel W, Crane BR (2016) Changes in active site histidine hydrogen bonding trigger cryptochrome activation. Proc Natl Acad Sci USA 113(36):10073-10078. https://doi.org/10.1073/pnas.1606610113

Harris S-RR, Henbest KB, Maeda K, Pannell JR, Timmel CR, Hore PJ, Okamoto H (2009) Effect of magnetic fields on cryptochromedependent responses in Arabidopsis thaliana. J R Soc Interface 6(41):1193-1205. https://doi.org/10.1098/rsif.2008.0519

Herbel V, Orth C, Wenzel R, Ahmad M, Bittl R, Batschauer A (2013) Lifetimes of Arabidopsis cryptochrome signaling states in vivo. Plant J 74(4):583-592. https://doi.org/10.1111/tpj.12144

Hong G, Pachter R (2015) Photoactivation of cryptochromes from Drosophila melanogaster and Sylvia borin: insight into the chemical compass mechanism by computational investigation. J Phys Chem B 119:3883-3892. https://doi.org/10.1021/jp508871h

Hore PJ, Mouritsen H (2016) The radical-pair mechanism of magnetoreception. Annu Rev Biophys 45:299-344. https://doi. org/10.1146/annurev-biophys-032116-094545
Hu Y, Vandenbussche F, Van der Straeten D (2017) Regulation of seedling growth by ethylene and the ethylene-auxin crosstalk. Planta 245(3):467-489. https://doi.org/10.1007/s00425-017-2651-6

Kattnig DR, Hore PJ (2017) The sensitivity of a radical pair compass magnetoreceptor can be significantly amplified by radical scavengers. Sci Rep 7:11640. https://doi.org/10.1038/s41598-017-09914 $-7$

Kattnig DR, Evans EW, Déjean V et al (2016) Chemical amplification of magnetic field effects relevant to avian magnetoreception. Nat Chem 8:384-391. https://doi.org/10.1038/nchem.2447

Kondoh M, Shiraishi C, Müller P, Ahmad M, Hitomi K, Getzoff ED, Terazima M (2011) Light-induced conformational changes in full-length Arabidopsis thaliana cryptochrome. J Mol Biol 413:128-137

Lin C, Top D, Manahan CC, Young MW, Crane BR (2018) Circadian clock activity of cryptochrome relies on tryptophan-mediated photoreduction. Proc Natl Acad Sci USA 115(15):3822-3827. https ://doi.org/10.1073/pnas.1719376115

Lüdemann G, Solov'yov IA, Kubař T, Elstner M (2015) Solvent driving force ensures fast formation of a persistent and well-separated radical pair in plant cryptochrome. J Am Chem Soc 137:1147-1156. https://doi.org/10.1021/ja510550g

Maeda K, Robinson AJ, Henbest KB, Hogben HJ, Biskup T, Ahmad M, Schleicher E, Weber S, Timmel CR, Hore PJ (2012) Magnetically sensitive light-induced reactions in cryptochrome are consistent with its proposed role as a magnetoreceptor. Proc Natl Acad Sci USA 109(13):4774-4779. https://doi.org/10.1073/pnas.11189 59109

Maffei ME (2014) Magnetic field effects on plant growth, development, and evolution. Front Plant Sci 5:445. https://doi.org/10.3389/ fpls.2014.00445

Marley R, Giachello CNG, Scrutton NS, Baines RA, Jones AR (2014) Cryptochrome-dependent magnetic field effect on seizure response in Drosophila larvae. Sci Rep 4:5799. https://doi. org/10.1038/srep05799

Mazzella MA, Cerdán PD, Staneloni RJ, Casal JJ (2001) Hierarchical coupling of phytochromes and cryptochromes reconciles stability and light modulation of Arabidopsis development. Development 128(12):2291-2299

Mouritsen H, Hore PJ (2012) The magnetic retina: light-dependent and trigeminal magnetoreception in migratory birds. Curr Opin Neurobiol 22:343-352

Müller P, Ahmad M (2011) Light-activated cryptochrome reacts with molecular oxygen to form a flavin-superoxide radical pair consistent with magnetoreception. J Biol Chem 286(24):21033-21040. https://doi.org/10.1074/jbc.m111.228940

Müller P, Yamamoto J, Martin R, Iwai S, Brettel K (2015) Discovery and functional analysis of a 4th electron-transferring tryptophan conserved exclusively in animal cryptochromes and (6-4) photolyases. Chem Commun 51:15502-15505. https://doi.org/10.1039/ c5 $5 \mathrm{cc} 06276 \mathrm{~d}$

Nießner C, Denzau S, Stapput K, Ahmad M, Peichl L, Wiltschko W, Wiltschko R (2013) Magnetoreception: activated cryptochrome 1a concurs with magnetic orientation in birds. J R Soc Interface 10(88):20130638. https://doi.org/10.1098/rsif.2013.0638

Nohr D, Franz S, Rodriguez R et al (2016) Extended electron-transfer in animal cryptochromes mediated by a tetrad of aromatic amino acids. Biophys J 111:301-311. https://doi.org/10.1016/j. bpj.2016.06.009

Nohr D, Paulus B, Rodriguez R et al (2017) Determination of radical-radical distances in light active proteins and their implication for biological magnetoreception. Angewandte Chemie Int Edn 56(29):8550-8554. https://doi.org/10.1002/anie.201700389

Occhipinti A, Santis A, Maffei ME (2014) Magnetoreception: an unavoidable step for plant evolution? Trends Plant Sci 19(1):1-4 
Ozturk N (2017) Phylogenetic and functional classification of the photolyase/cryptochrome family. J Photochem Photobiol 93(1):104111. https://doi.org/10.1111/php.12676

Procopio M, Link J, Engle D, Witczak J, Ritz T, Ahmad M (2016) Kinetic modeling of the Arabidopsis cryptochrome photocycle: $\mathrm{FADH}^{\mathrm{o}}$ accumulation correlates with biological activity. Front Plant Sci 7:888. https://doi.org/10.3389/fpls.2016.00888

Qin S, Yin H, Yang C, Dou Y, Liu Z, Zhang P et al (2016) A magnetic protein biocompass. Nat Mater 15:217-226. https://doi. org/10.1038/nmat4484

Ritz T, Adem S, Schulten K (2000) A model for photoreceptor-based magnetoreception in birds. Biophys J 78:707-718. https://doi. org/10.1016/S0006-3495(00)76629-X

Ritz T, Yoshii T, Foerster C, Ahmad M (2010) Cryptochrome: a photoreceptor with the properties of a magnetoreceptor? Commun Integr Biol 3(1):24-27. https://doi.org/10.4161/cib.3.1.9865

Rodgers CT (2009) Magnetic field effects in chemical systems. Pure Appl Chem 81(1):19-43. https://doi.org/10.1351/ PAC-CON-08-10-18

Schulten K, Staerk H, Weller A, Werner H-J, Nickel B (1976) Magnetic field dependence of the geminate recombination of radical ion pairs in polar solvents. Z Phys Chem 101:371-390

Shalitin D, Yu X, Maymon M, Mockler T, Lin C (2003) Blue lightdependent in vivo and in vitro phosphorylation of Arabidopsis cryptochrome 1. Plant Cell 15(10):2421-2429. https://doi. org/10.1105/tpc.013011

Sheppard D, Li J, Henbest K et al (2017) Millitesla magnetic field effects on the photocycle of an animal cryptochrome. Sci Rep 7:42228. https://doi.org/10.1038/srep42228

Solov'yov IA, Chandler DE, Schulten K (2007) Magnetic field effects in Arabidopsis thaliana cryptochrome-1. Biophys J 92(8):27112726. https://doi.org/10.1529/biophysj.106.097139

Tan ST, Dai C, Liu HT, Cell X-HW (2013) Arabidopsis casein kinase1 proteins CK1. 3 and CK1. 4 phosphorylate cryptochrome 2 to regulate blue light signaling. Plant Cell 25:2618-2632. https://doi. org/10.1105/tpc. 113.114322

Thöing C, Oldemeyer S, Kottke T (2015) Microsecond deprotonation of aspartic acid and response of the $\alpha / \beta$ subdomain precede C-terminal signaling in the blue light sensor plant cryptochrome. J Am Chem Soc 137:5990-5999. https://doi.org/10.1021/jacs.5b01404

Van der Straeten J, Gailly P, Malkemper P (2018) Low-light dependence of the magnetic field effect on cryptochromes: possible relevance to plant ecology. Front Plant Sci 9:121. https://doi. org/10.3389/fpls.2018.00121

Wang Q, Barshop WD, Bian M, Vashisht AA et al (2015) The blue light-dependent phosphorylation of the $\mathrm{CCE}$ domain determines the photosensitivity of Arabidopsis CRY2. Mol Plant 8:631-643

Wiltschko R, Wiltschko W (2012) Magnetoreception. Adv Exp Med Biol 739:126-141. https://doi.org/10.1007/978-1-4614-1704-0_8

Wiltschko R, Wiltschko W (2014) Sensing magnetic directions in birds: radical pair processes involving cryptochrome. Biosensors 4(3):221-242. https://doi.org/10.3390/bios4030221

Wiltschko R, Ahmad M, Nießner C, Gehring D, Wiltschko W (2016) Light-dependent magnetoreception in birds: the crucial step occurs in the dark. J R Soc Interface 13:20151010. https://doi. org/10.1098/rsif.2015.1010

Worster S, Kattnig DR, Hore PJ (2016) Spin relaxation of radicals in cryptochrome and its role in avian magnetoreception. J Chem Phys 145:035104. https://doi.org/10.1063/1.4958624

Wu CL, Fu TF, Chiang MH, Chang YW, Her JL, Wu T (2016) Magnetoreception regulates male courtship activity in Drosophila. PLoS One 11(5):e0155942. https://doi.org/10.1371/journal.pone.01559 42

Xu C, Yin X, Lv Y, Wu C, Zhang Y, Song T (2012) A near-null magnetic field affects cryptochrome-related hypocotyl growth and flowering in Arabidopsis. Adv Space Res 49(5):834-840. https ://doi.org/10.1016/j.asr.2011.12.004

Xu C, Lv Y, Chen C, Zhang Y, Wei S (2014) Blue light-dependent phosphorylations of cryptochromes are affected by magnetic fields in Arabidopsis. Adv Space Res 53(7):1118-1124

Xu C, Li Y, Yu Y, Zhang Y, Wei S (2015) Suppression of Arabidopsis flowering by near-null magnetic field is affected by light. Bioelectromagnetics 36(6):476-479. https://doi.org/10.1002/bem.21927

Yang Z, Liu B, Su J, Liao J, Lin C, Oka Y (2017) Cryptochromes orchestrate transcription regulation of diverse blue light responses in plants. Photochem Photobiol 93(1):112-127. https://doi. org/10.1111/php.12663

Yoshii T, Ahmad M, Helfrich-Förster C (2009) Cryptochrome mediates light-dependent magnetosensitivity of Drosophila's circadian clock. PLoS Biol 7(4):e1000086. https://doi.org/10.1371/journ al.pbio. 1000086 